\title{
Assessment of Blockage effects in Wind Tunnel Testing of Wind Turbines
}

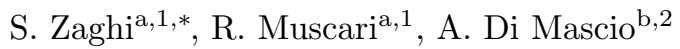 \\ ${ }^{a}$ CNR-INSEAN, Istituto Nazionale per Studi ed Esperienze di Architettura Navale, Via di \\ Vallerano 139, Rome, Italy, 00128 \\ ${ }^{b}$ CNR-IAC, Istituto per le Applicazioni del Calcolo "M. Picone", Via dei Taurini 19, Rome, \\ Italy, 00185
}

\begin{abstract}
The aim of the present paper is the analysis of simplified boundary conditions to be used in numerical simulations, to take into account blockage effects for wind tunnel experiments of large scale wind turbines. The goal is the development of an efficient and reliable tool to be used to correct data obtained from experiments where the blockage coefficient is high and/or the turbine is highly loaded, for which traditional correction coefficients (derived from the Glauert theory or its more recent versions) fail.
\end{abstract}

Numerical simulations of the flow around a three-bladed model-scale wind turbine with horizontal axis are reported; in all test cases, the turbine diameter is comparable with test section dimensions, and therefore blockage effects are significant. The actual experiments were approximated numerically with a simplified wind tunnel geometry, that retains the symmetries of the isolated turbine simulation in a rotating frame and therefore allows steady state computations. To this end, two circular wind tunnel were tested: for the first, the radius was chosen to retain the same cross section as the actual wind tunnel; in the second, its was set to be equal to half of the smallest cross section dimension.

The aerodynamic performances of the turbine, in terms of power and thrust coefficients, are analyzed and compared with available experimental data. Detailed analysis of the flow in the wake is also reported. Analogous simulations

\footnotetext{
* Corresponding author

Email addresses: s.zaghi@insean.it (S. Zaghi), roberto.muscari@cnr.it (R. Muscari), a.dimascio@iac.cnr.it (A. Di Mascio)

${ }^{1}$ Ph. D., Aerospace Engineer, Research Scientist at CNR-INSEAN.

${ }^{2}$ Ph. D., Aerospace Engineer, Research Scientist at CNR-IAC.
} 
in an unbounded domain are also reported.

Keywords: Wind Energy, Wind Turbine, Wind Turbine Wake, Blockage

\section{Introduction}

Nowadays, research on renewable energy is strongly increasing because of both the increased awareness of the need to preserve the environment and the decreased availability of fossil fuels. In the course of time, wind turbines evolved from rather primitive and small windmills to technologically advanced and huge mechanical systems. Their efficiency has also fostered the deployment of large Wind Turbine Farms (WTF). A historical review of wind energy conversion, together with a review of aerodynamic modelling and optimization of wind turbines, can be found in Sørensen [1].

Of course, the larger the turbine, the more demanding is the design of the apparatus, as many aerodynamic, structural and environmental problems arises. Moreover, in large wind farms, the downstream turbine always operate in offdesign conditions, and therefore the accurate prediction of the Horizontal-Axis Wind Turbine (HAWT) wake is of paramount importance.

The increasing importance of wind energy is naturally accompanied by an intense design activity by numerical simulation, in particular for new concept designs. Of course, the development of new apparatuses requires accurate experimental confirmation, that fostered many experimental campaigns about turbine performances. The most comprehensive and recent measurements were carried out in the large wind tunnel test at NASA-Ames in the framework of the NREL Phase VI Wind Tunnel project [2], in the experimental campaign in the framework of the international cooperation project "Mexico" $[3,4]$ and in the experiments performed at the Norwegian University of Science and Technology (NTNU) [5, 6]. The wind tunnels adopted in the first two experiments were very large when compared with turbine diameter, and therefore blockage effects were estimated negligible; in the third one, instead, the presence of the side walls could not be simply neglected, because the ratio of the rotor area to the test section area is about $12.4 \%[5]$.

There are many methods to assess blockage effects, that must be properly estimated to extract the required information in the design phase. They range 
from the traditional methods based on the momentum theory (like the one developer by Glauert [7] or its recent versions $[8,9,10])$ to detailed unsteady Navier-Stokes simulations that take into account the actual wind tunnel geometry. The first kind of corrections are very useful when the blockage coefficient is small and when the turbine is lightly or mildly loaded. On the contrary, when studying large (when compared to tunnel size) turbines, possibly highly loaded, a trustworthy answer about blockage effects can be obtained only by full Navier-Stokes simulations. This last approach, although reliable, is nevertheless expensive, because the flow cannot be steady, even when simplifying the problem by considering an isolated turbine in the rotating frame, as in general tunnel walls are not axisymmetric.

In the present paper, we investigated the possibility to replace the actual geometry of the wind tunnel with a circular section, in order to perform steady state computations in the rotating frame, while retaining flow details around the actual turbine geometry in the full range of loading conditions. This approach is much faster than the full unsteady Navier-Stokes simulation with the actual geometry, and therefore allows to widen the range of test cases for fixed computational resources. Of course, the choice of the equivalent wind tunnel section is all but trivial, as the only parameter at disposal (the section radius) must be such that the outcome of the simulations match both global and local quantities with the actual experiments. To this purpose, two test sections were considered: in the first, the radius was chosen so that the fictitious section has the same area as the actual section; in the second, the radius was set equal to the half of the smallest dimension of the cross section. Numerical results were checked against the data obtained in the NTNU experiment $[11,5,12]$.

The paper is organized as follows: a brief description of the model-scale wind turbine is reported in section 2; mathematical and numerical models are briefly recalled in section 3; details of the numerical setups are explained in 4; discussion on the numerical results is contained in 5; concluding remarks can be found in section 6 . 


\section{Wind turbine geometry}

The wind turbine considered in the present paper is the three-bladed horizontal axis turbine, with NREL S826 section profile [13], designed and manufactured at the NTNU [5]. The profile has $14 \%$ thickness, designed to optimize the performances for a Reynolds number of $2 \times 10^{6}$. The blades are made of aluminium and mounted on a small hub (nacelle) with a diameter $d=0.09 \mathrm{~m}$. Between the blade and the nacelle there is a transition surface, i.e. a linear interpolation between the S826 profile and the circular profile on the nacelle surface. Figure 1a shows two side-views, whereas figure 1b summarizes its main characteristics. Note that the blade terminates with a sharp cut.

More details about the turbine geometry (tabulated coordinates of blade profiles, twist angle and chord length) can be found in [5], where we also took all experimental data used to validate our numerical results.

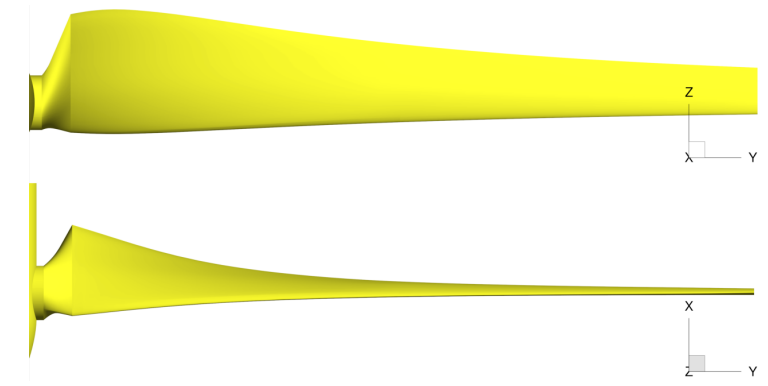

(a) Blade profiles (b) Main characteristics

\begin{tabular}{|c|c|}
\hline Number of Blades & 3 \\
\hline ROTOR DIAMETER $[\mathrm{m}]$ & 0.9000 \\
\hline NACELLE DiAMETER $[\mathrm{m}]$ & 0.0900 \\
\hline ROOT CHORD $[m]$ & 0.0495 \\
\hline TIP CHORD $[m]$ & 0.0259 \\
\hline RoOT TWIST ANGLE $[\mathrm{deg}]$ & 38.00 \\
\hline TIP TWIST ANGLE $[\mathrm{deg}]$ & -0.72 \\
\hline
\end{tabular}

Profile thickness $[\%$ chord $] \quad 14.0$

Figure 1: Model-scale blade geometry

\section{Mathematical and Numerical Models}

The presented simulations have been performed by means an in-house software developed and validated for aero/hydrodynamics problems, see [14, 15, 16 , 17]. The mathematical model is based on the integration of the Reynolds Averaged Navier-Stokes equations (RANS) written in non-dimensional form in the frame of reference fixed to the turbine [18]. Several closure models are available in the code for computing the eddy viscosity; in the present work, the Spalart and Allmaras one-equation model [19] has been adopted for all simulations. The equations are discretized by means of a conservative cell-centered finite volume 
approach, on a multi-block structured body-fitted grid with partial overlapping. Several approximation schemes are available in the code for convective fluxes, from second to fourth order accuracy [16]. Diffusive fluxes are always computed by centered second order approximation. The scheme being fully implicit in time, the resulting non-linear algebraic system of equations is solved iteratively by a dual (or pseudo) time approach [20], full multi-grid iteration [21] and the implicit approximate factorization technique [22] with local time stepping.

The problem is closed by enforcing appropriate conditions at physical and computational boundaries. On solid walls, the relative velocity is set to zero (whereas no condition on the pressure is required); at the (fictitious) inflow boundary, velocity is set to the undisturbed flow value, and the pressure is extrapolated from inside; on the contrary, the pressure is set to the undisturbed free-stream value at the outflow, whereas velocity is extrapolated from inner points. The initial conditions for all the simulations are set to the uniform free stream conditions.

The simulation code is parallelized to run on both distributed and shared memory machines.

Table 1: Inflow conditions and reference values

(a) Inflow conditions

\begin{tabular}{ll}
\hline$U_{\text {inf }}\left[\frac{\mathrm{m}}{\mathrm{s}}\right]$ & 10.00 \\
$\rho_{\text {inf }}\left[\frac{\mathrm{kg}}{\mathrm{m}^{3}}\right]$ & 1.20 \\
$\nu_{\text {inf }}\left[\frac{\mathrm{m}^{2}}{\mathrm{~s}}\right]$ & $1.50 \cdot 10^{-5}$ \\
\hline
\end{tabular}

(b) Reference values

\begin{tabular}{ll}
\hline$U_{0}$ & $0.7 \lambda U_{\text {inf }}=0.7 \omega R$ \\
$\rho_{0}\left[\frac{\mathrm{kg}}{\mathrm{m}^{3}}\right]$ & $1.200=\rho_{\text {inf }}$ \\
$\nu_{0}\left[\frac{\mathrm{m}^{2}}{\mathrm{~s}}\right]$ & $1.500 \cdot 10^{-5}=\nu_{\text {inf }}$ \\
$L_{0}[\mathrm{~m}]$ & $25.926 \cdot 10^{-3}=$ Chord $_{\text {tip }}$ \\
\hline
\end{tabular}

Table 2: Tests matrix

\begin{tabular}{ccc}
\hline$\lambda$ & $\omega\left[\frac{\mathrm{rad}}{\mathrm{s}}\right]$ & $R e$ \\
\hline 1 & 22.222 & $1.210 \cdot 10^{4}$ \\
2 & 44.444 & $2.420 \cdot 10^{4}$ \\
3 & 66.667 & $3.630 \cdot 10^{4}$ \\
4 & 88.889 & $4.840 \cdot 10^{4}$ \\
5 & 111.111 & $6.049 \cdot 10^{4}$ \\
6 & 133.333 & $7.259 \cdot 10^{4}$ \\
\hline
\end{tabular}

\begin{tabular}{ccc}
\hline$\lambda$ & $\omega\left[\frac{\mathrm{rad}}{s}\right]$ & $\operatorname{Re}$ \\
\hline 7 & 155.556 & $8.469 \cdot 10^{4}$ \\
8 & 177.778 & $9.679 \cdot 10^{4}$ \\
9 & 200.000 & $1.089 \cdot 10^{5}$ \\
10 & 222.222 & $1.210 \cdot 10^{5}$ \\
11 & 244.444 & $1.331 \cdot 10^{5}$ \\
12 & 266.667 & $1.452 \cdot 10^{5}$ \\
\hline
\end{tabular}




\section{Numerical Setups}

Tables $1 \mathrm{a}$ and $1 \mathrm{~b}$ report the test conditions and the reference values used to render all the quantities non-dimensional. The inflow free-stream is supposed to be uniform with zero background turbulence and aligned to the turbine axis. These conditions mimic the NTNU experiments, where a turbulence intensity of about $T I=0.3 \%$ at the inlet to the test section was measured (see [23, 24]).

As already said, the turbine tower has been always neglected, in order to compute a steady solution in the rotating frame of reference.

Table 2 reports the test matrix, where $\lambda=(\omega R) / U_{\text {inf }}$ is the tip speed ratio and $R e=\left(U_{0} L_{0}\right) / \nu$ is the Reynolds number, with $L_{0}$ the profile chord at the tip and $U_{0}=0.7 \lambda U_{i n f}$. The design condition is $\lambda=6$, corresponding to the maximum output power. This regime corresponds to a frequency $f=21.220 \mathrm{~Hz}$, i.e. to a period $t=1 / f=0.047 \mathrm{~s}$.

Two simulation campaigns were done to assess the likelihood of the equivalent circular wind tunnel sections, whereas a third one was carried out to compute the unbounded flow and to quantify the need for a correction to the experimental data. For each campaign the test matrix is the same and it is reported in table 2. For the computation in the bounded domain, free slip conditions were enforced on the tunnel walls.

Table 3 reports the main dimensions of the numerical grids used. Grid dimensions are expressed in millions of finite volumes. The grid resolution in the boundary layer cells has been fixed, on the basis of highest value for Reynolds number in table 2 , in such a way that the distance from the wall of the first grid cell in terms of wall coordinates $y^{+}$is always smaller than 1 and there are at least 32 finite volumes in the boundary layer thickness. The grid of the blade surface has 150 cells in spanwise direction and 200 in chordwise direction.

Table 3: Numerical grids dimensions

\begin{tabular}{lcr}
\hline REgion & Number of Blocks & Number of Finite volumes \\
\hline Background & 142 & $3.52 \mathrm{M}$ \\
Single Blade & $78(\times 3=234)$ & $2.50 \mathrm{M}(\times 3=7.50 M)$ \\
Nacelle & 40 & $1.40 \mathrm{M}$ \\
\cline { 2 - 3 } Total & 416 & $12.42 \mathrm{M}$ \\
\hline
\end{tabular}


The grid is made of many patched and partially overlapped structured blocks. The background consists of different groups of toroidal blocks, while the blocks closer to the blades were built to obtain fine resolution of the wake close to the tip and to the root. The blocks around the blade were tailored to accurately fit the surface.

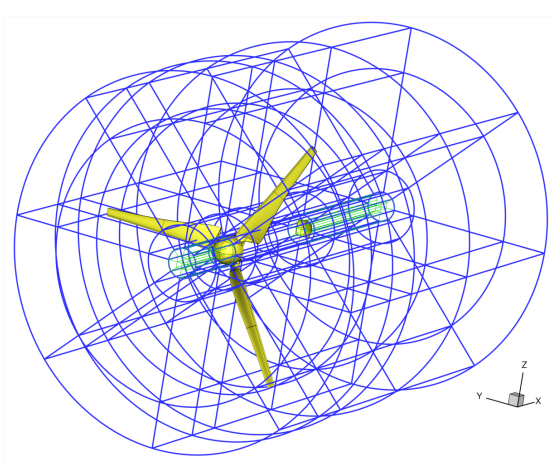

(a) Near field background topology

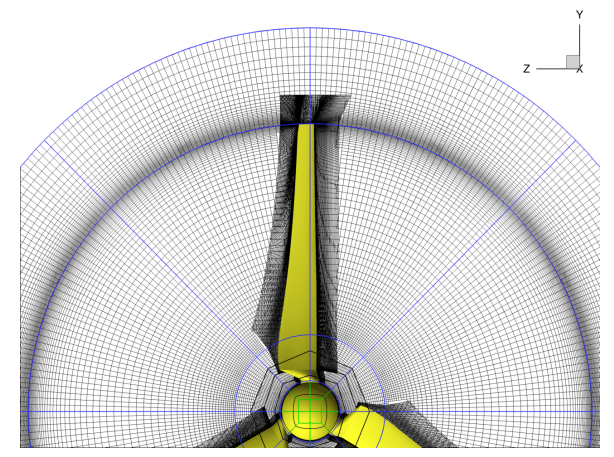

(c) Cross slice mesh detail

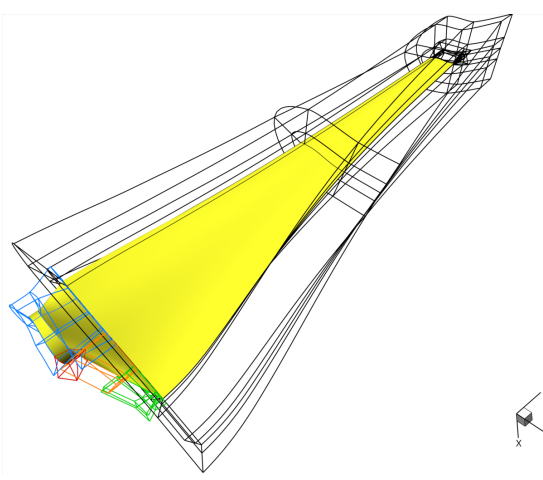

(b) Blade topology

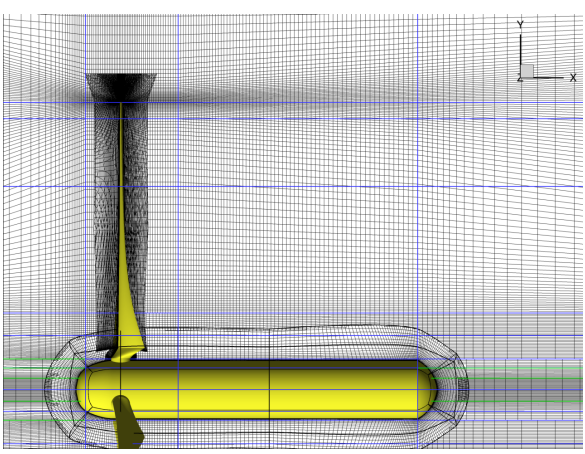

(d) Longitudinal slice mesh detail

Figure 2: Mesh overview. Yellow: solid walls

Figure 2 shows an overview of the mesh for the unbounded simulations. Note that only the near field background topology has been reported, as the far field boundaries have been placed far enough not to affect the solution; in particular, the boundary is $14 R$ far, where $R$ is the turbine radius. The inflow boundary is placed $8 R$ upstream the turbine, while the outflow boundary condition is placed $14 R$ downstream.

As already said, the simulations with uniform flow conditions have been done in a rotating frame of reference, and the whole nacelle is supposed to rotate rigidly with the blades. This is of course an approximation, as a portion 
of the nacelle is fixed to the pylon. This simplified condition is consistent with the fact that the whole pylon was removed, in order to simplify and speed up the computation.

The second and the third simulations campaigns have been aimed to assess the effect of the wind tunnel walls. The test section of the experimental wind tunnel is $H=1.9 \mathrm{~m}$ high and $L=2.7 \mathrm{~m}$ wide (see Krogstad and Adaramola [5]), the test section area being $A_{\text {exp }}=5.13 \mathrm{~m}^{2}$.

In the first campaign with bounded domain, the radius of the equivalent section was chosen equal to $R_{e q}=$ $\sqrt{A_{\text {exp }} / \pi}=1.278 m \cong 2.84 R$. For this value of $R_{e q}$, the test section area remains the same as the actual wind tunnel, and therefore this choice reproduce

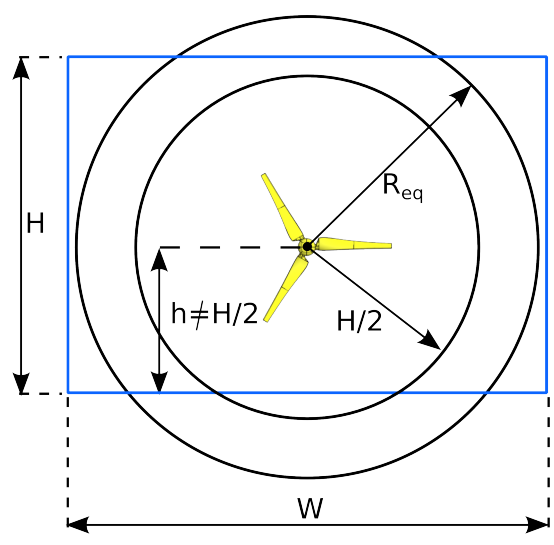

Figure 3: Actual and equivalent test sections. Blue rectangle: actual section; black circles: equivalent sections; $H=1.9 \mathrm{~m}$, $W=2.7 m, h=0.817 m, R_{e q}=1.278 m$ a situation that maintains the blockage ratio (i.e., the ratio between the rotor swept area and the wind tunnel cross-sectional area) constant.

In the second simulation set with walls, the equivalent radius $R_{H / 2}=0.95 \mathrm{~m}$ was set to half the smallest cross section dimension. For this arrangement, the blockage coefficient is larger than the actual one, but the hindrance to wake development is closer to the one due to the real geometric constraint (see figure 3). The numerical grids used for these two last campaigns are almost identical to the one for the simulations without walls, the only difference being the position of the outer boundaries.

\section{Results and discussion}

\subsection{Verification}

In all the simulations, numerical uncertainty was assessed by computing the solution on 3 grid levels, each obtained from the next finer level by removing every other point in all coordinate directions (i.e. the grid levels ratio is always 2). Tables 4a and 4b summarize convergence analysis; here $S_{l}$ with $l=c, m, f$ are 
the non-dimensional power and thrust coefficients computed on the finest $(f)$, medium $(m)$ and coarse $(c)$ grids. The tables report the estimated uncertainties $U$ and convergence order $\sigma$ evaluated by means of Richardson's extrapolation (see, for instance, Roache [25]). All the simulations were stopped when the maximum non-dimensional residual (in most cases, the residual of the axial momentum) dropped below $10^{-4}$. The number of iteration required was always $\sim 5000$. The estimated uncertainty was found to be lower than $2.1 \%$ for all conditions.

Table 4: Convergence analysis, $\lambda=6$

(a) Power Coefficient, $S=C_{P}$

\begin{tabular}{lccccc}
\hline & $S_{c}$ & $S_{m}$ & $S_{f}$ & $\sigma$ & $\mathrm{U} \%$ \\
\hline No WT & $2.620 \cdot 10^{-1}$ & $3.681 \cdot 10^{-1}$ & $3.931 \cdot 10^{-1}$ & 2.09 & 2.08 \\
\hline$R=R_{e q}$ & $3.560 \cdot 10^{-1}$ & $4.350 \cdot 10^{-1}$ & $4.597 \cdot 10^{-1}$ & 1.68 & 1.76 \\
\hline$R=H / 2$ & $3.924 \cdot 10^{-1}$ & $4.809 \cdot 10^{-1}$ & $5.090 \cdot 10^{-1}$ & 1.65 & 1.81
\end{tabular}

(b) Thrust Coefficient, $S=C_{T}$

\begin{tabular}{lccccc}
\hline & $S_{c}$ & $S_{m}$ & $S_{f}$ & $\sigma$ & $\mathrm{U} \%$ \\
\hline No WT & $7.306 \cdot 10^{-1}$ & $7.599 \cdot 10^{-1}$ & $7.781 \cdot 10^{-1}$ & 0.69 & 0.77 \\
\hline$R=R_{e q}$ & $8.174 \cdot 10^{-1}$ & $8.350 \cdot 10^{-1}$ & $8.406 \cdot 10^{-1}$ & 1.63 & 0.22 \\
\hline$R=H / 2$ & $8.501 \cdot 10^{-1}$ & $8.733 \cdot 10^{-1}$ & $8.812 \cdot 10^{-1}$ & 1.55 & 0.30 \\
\hline
\end{tabular}

\subsection{Performance analysis}

Figures 4 and 5 report the comparison of computed power and thrust coefficients

$$
C_{P}=\frac{P}{\frac{1}{2} \rho_{i n f} U_{i n f}^{3} A} \quad C_{T}=\frac{T}{\frac{1}{2} \rho_{i n f} U_{i n f}^{2} A}
$$

( $P=M_{x} \cdot \omega$ being the extracted power, $M_{x}$ the axial moment, $T$ the axial force and $A$ the rotor area), with experiments $[11,5]$.

It can be seen that good agreement with experiments is always achieved when the rotational speed $(\lambda)$ is low. For increasing speed $(\lambda>4)$, instead, the predictions in unbounded domain significantly depart from the experimental data, and therefore a correction is required. The best agreement for simulation with bounded domains is observed for the power coefficient when the computation includes the fictitious wind tunnel with $R=R_{e q}$, in the whole range of 
$\lambda$. When comparing the thrust coefficient, instead, it seems that the agreement is better when the walls of the equivalent wind tunnel are placed at $R=H / 2$. In the numerical results shown in figures 4 and 5, the pressure (Eulerian) and viscous terms are reported separately. As expected, the contribution of the viscous stresses to the overall thrust is negligible, whereas they play a major role in the evaluation of the power coefficient, particularly for higher values of $\lambda$. However, also for $C_{p}$, blockage affects only on the Eulerian part, as the viscous part remains practically the same for any wall distance.

From the reported results, it can be seen that, although the complete match with experiments is not attained in neither of the two arrangements, the presence of a lateral constrain largely improves the agreement with data, and the best choice to estimate blockage on global performances seems to be $R_{e q}$.

\subsection{Flow field}

An idea of the wake structure for $\lambda=[3,6,9]$ is reported in figure 6 , where the tip vortices are identified by the well-known Jeong's criterion [26] ( $\sigma_{2}$ criterion). The reported iso-surfaces are colored with pressure contours.

It can be seen that, for small $\lambda$, tip vortices are twisted in a helical structure with large pitch (because of the low angular velocity), whereas the blade root generates vortices that soon merge with those produced by the nacelle. In this condition, large separation zones appear on the blade suction side (see figure 7a). The coils of tip vortices became more and more compact as $\lambda$ increases and blades root vorticity becomes distinguishable from the one generated by the nacelle. The large separation zones completely disappear for higher angular velocity, and this has a marked effect on the curves of thrust and, in particular, power coefficients (figures 4 and 5) that exhibit a sudden increase of steepness between $\lambda=3$ and $\lambda=4$. The growth of the slipstream (the turbines slows down the free stream) can be clearly observed, together with a delay in pressure recovery in the tip vortices.

For medium and high values of $\lambda(\lambda \geq 6)$, the vortices cluster toward the turbine and disappear at a very short distance downstream. This is probably due to the poor performances of the RANS model adopted, unable to properly describe flows with intense vortical structures far from walls. For a complete description of vortex evolution in the far wake, a Detached Eddy Simulation 


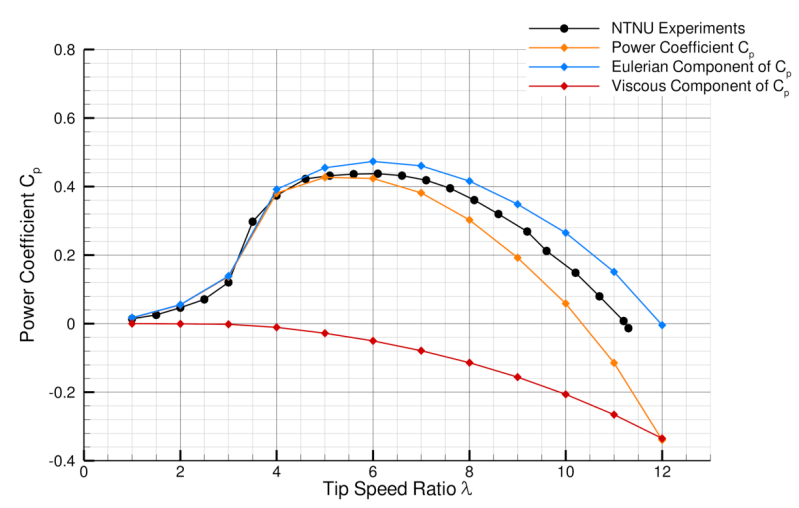

(a) Unbounded domain

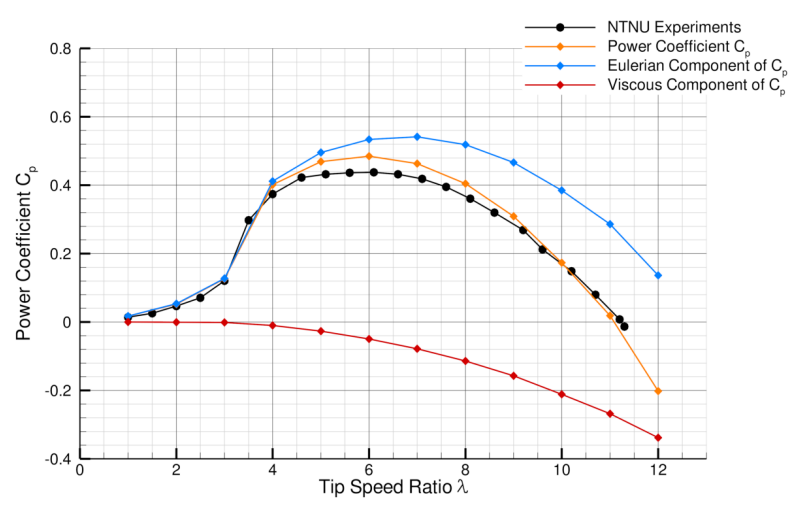

(b) Wind tunnel walls at $R_{e q}$

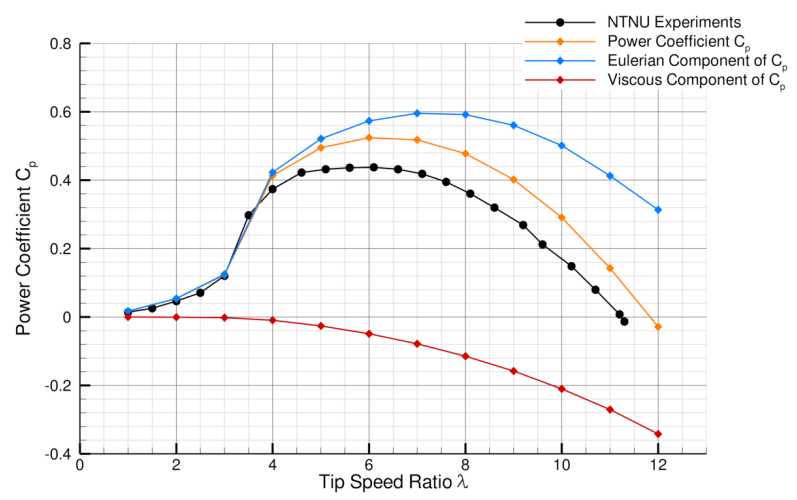

(c) Wind tunnel walls at $\frac{H}{2}$

Figure 4: Power $C_{P}$ coefficient comparison: experiments vs numerical results without and with wind tunnel walls 


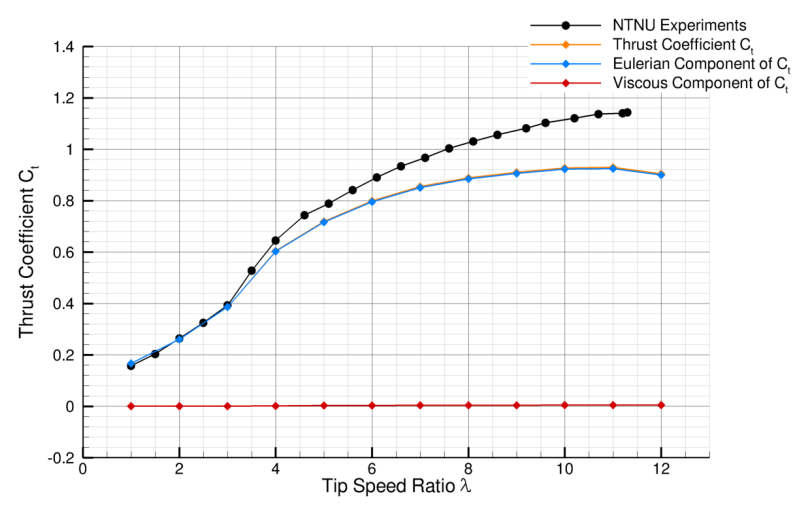

(a) Unbounded domain

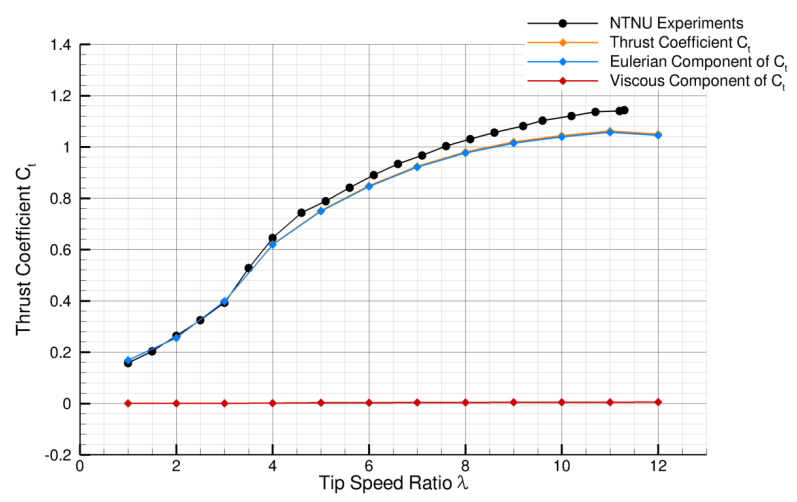

(b) Wind tunnel walls at $R_{e q}$

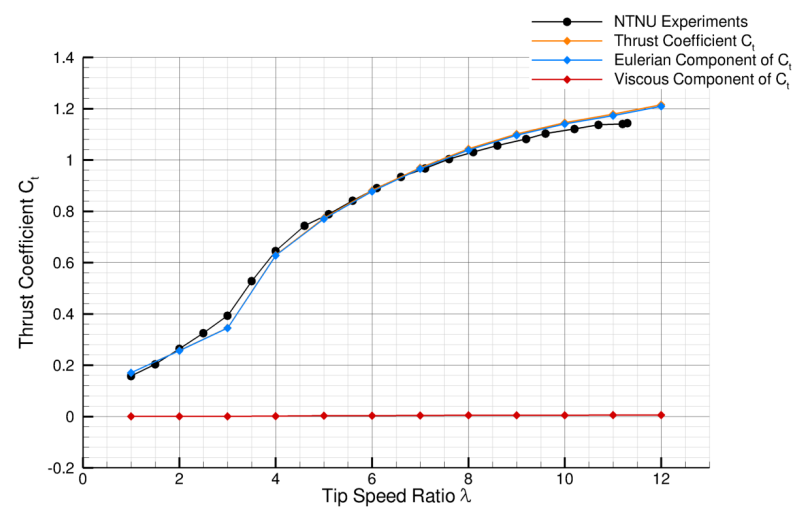

(c) Wind tunnel walls at $\frac{H}{2}$

Figure 5: Thrust $C_{T}$ coefficient comparison: experiments vs numerical results without and with wind tunnel walls 


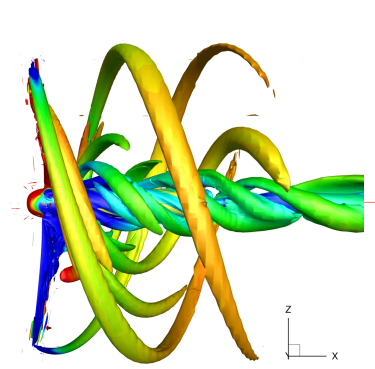

(a) $\lambda=3$, No WT

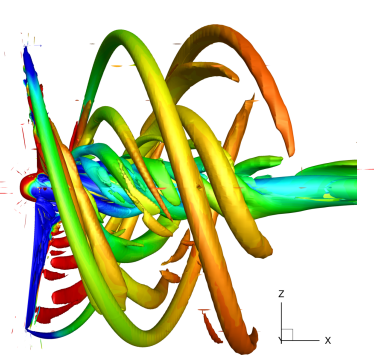

(b) $\lambda=3, R=R_{e q}$

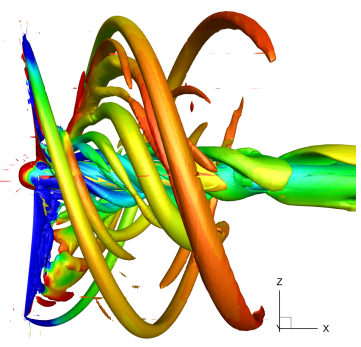

(c) $\lambda=3, R=H / 2$

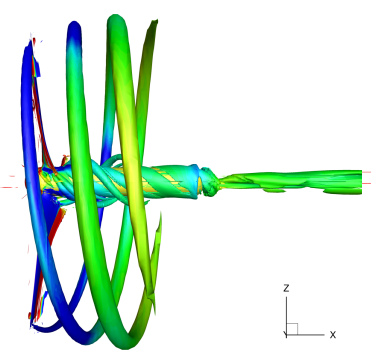

(d) $\lambda=6$, No WT

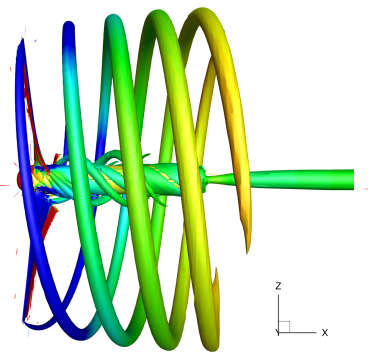

(e) $\lambda=6, R=R_{e q}$

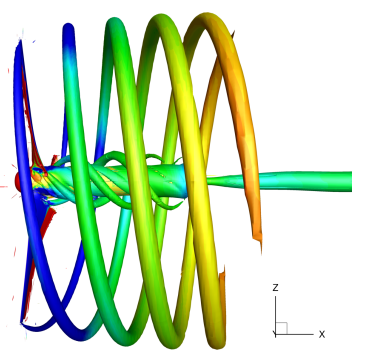

(f) $\lambda=6, R=H / 2$

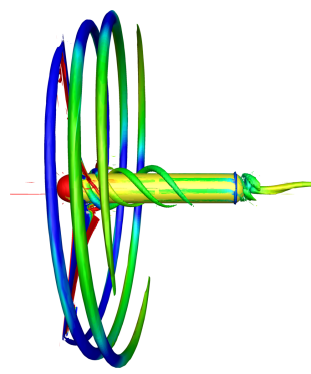

(g) $\lambda=9$, No WT

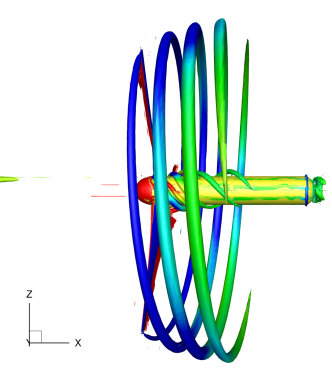

(h) $\lambda=9, R=R_{e q}$

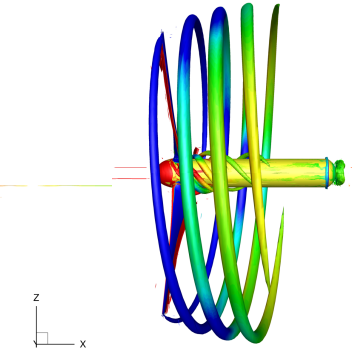

(i) $\lambda=9, R=H / 2$

Figure 6: Vortices cores colored by non-dimensional pressure levels, $\frac{\sigma_{2}}{f^{2}}=-8 ; \lambda \in[3,6,9]$

(DES) is better suited to the task, as shown in [27, 28] for propeller wake. However, a DES is intrinsically unsteady, also in the rotating reference frame, an this would render the computation far less efficient. Nevertheless, in [27, 28] is also proved that the results obtained with RANS and DES, performed with the models in $[29,30]$, perfectly match in terms of global coefficients and in the near wake, and therefore a (steady) RANS simulation is up to the task for the present investigation.

Figures 8 and 9 report a quantitative analysis of the wake features for the 


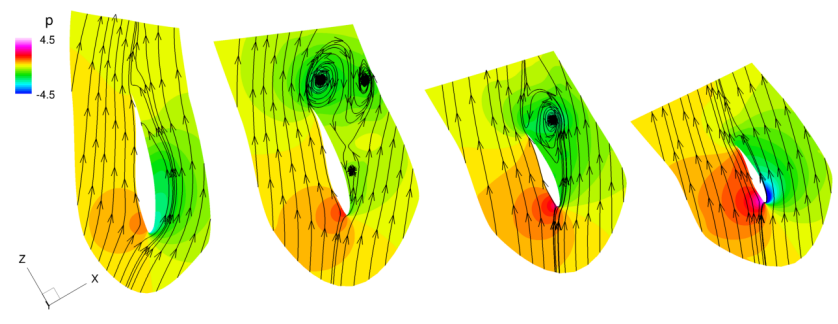

(a) $\lambda=3$

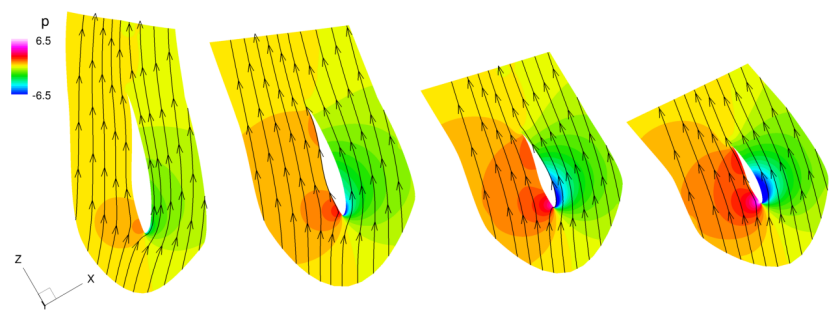

(b) $\lambda=4$

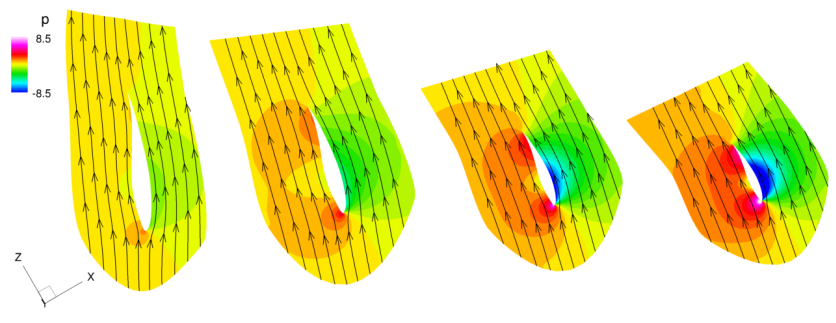

(c) $\lambda=6$

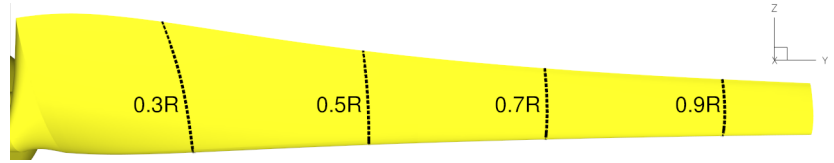

(d) Radius of the radial slices

Figure 7: Pressure contours and streamlines on radial slices

three numerical campaigns. The slipstream diameter and pitch were computed by tracking the radial position of the tip vortex cores, identified by the $\sigma_{2}$ criterion. As expected, figure 8 shows that the pitch increases when moving the wall closer to the slipstream. This can be justified by observing that, when the cross section reduces, the slipstream is contracted and therefore the average velocity within the wake increases, for a given flow rate. As the pitch can be estimated approximately by $\sim U_{\text {averaged }} \omega / R_{\text {slipstream }}$, its increase can be attributed to both the growth of the average speed in the wake $U_{\text {averaged }}$ and to the reduction in the slipstream radius $R_{\text {slipstream }}$. 


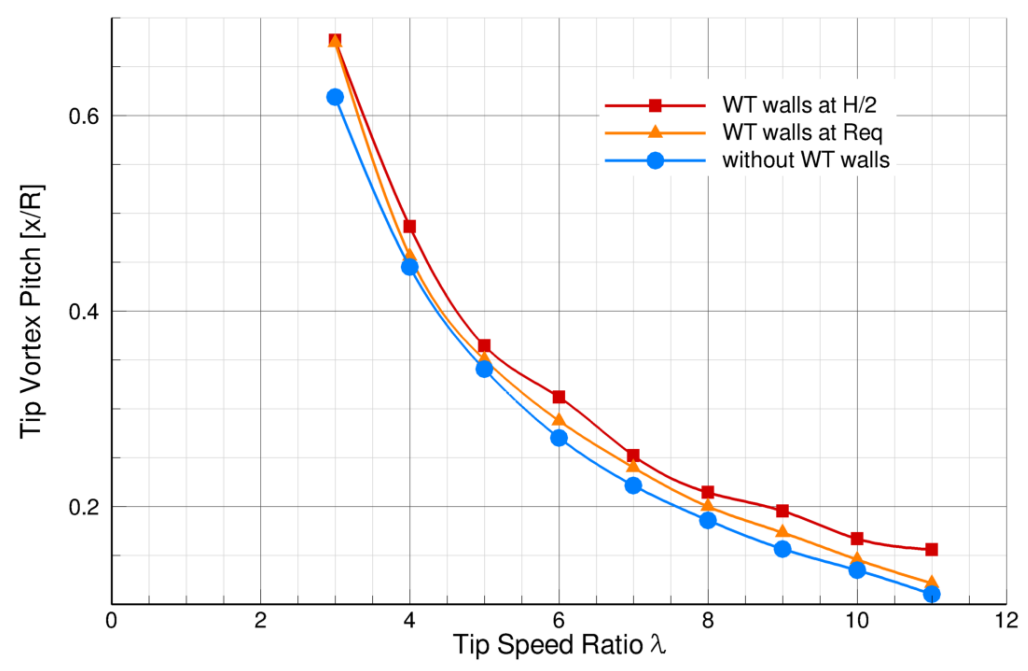

Figure 8: Tip Vortex Pitch

Figure 9 reports the slipstream profiles for three loading conditions. As for the pitch, the axial development of the wake considerably depends on the position of the side walls. Unfortunately, we lack experimental information about this details, but also in view of what shown in the next section, probably the correct prediction is the one with $R_{H / 2}$.

\subsection{Velocities Profiles}

More interesting (given the goal of the present paper) is the comparison of computed velocity profiles with measurements, reported in figure 10, where the averaged axial velocity profiles at three positions downstream the turbine, specifically at 1, 3 and 5 diameter downstream, are shown. Of course, in the region where the effects of the turbine tower wake is relevant (around $y / R=0$ ), none of the simulations gives good results, it being completely removed from the computations. Moreover, the simulations show a strong velocity defect (not present in the experiments) caused by an over-elongated recirculating zone behind the nacelle. Far from this central region, it can be clearly seen that the simulation that ignores wind tunnel walls fails to give the experimental value, that clearly suffers from blockage effects, of the axial velocity out of the slipstream. 


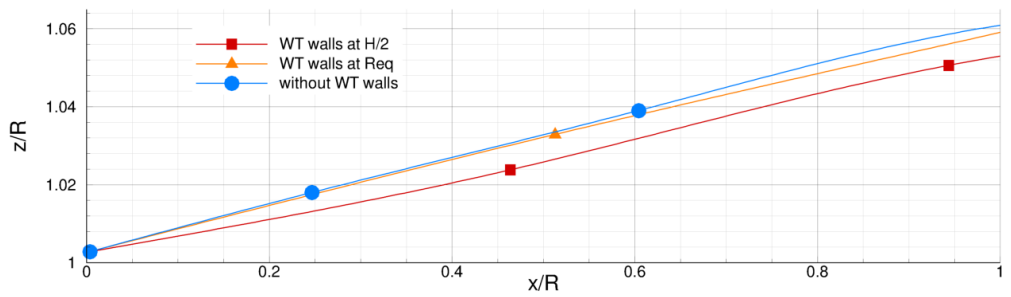

(a) $\lambda=3$

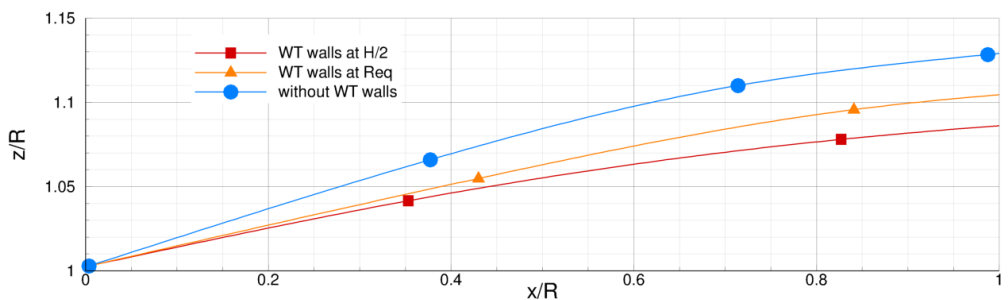

(b) $\lambda=6$

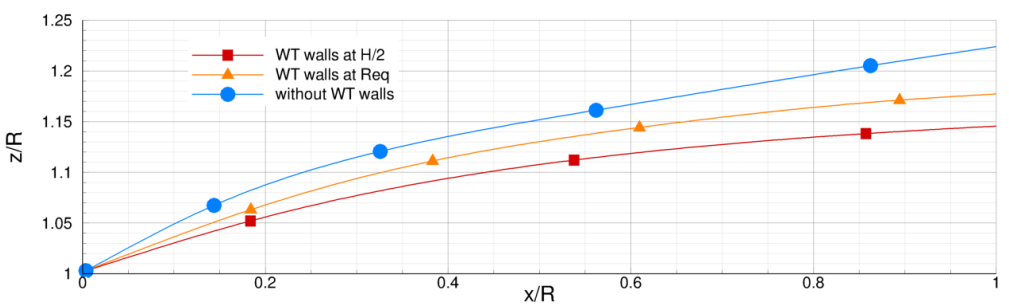

(c) $\lambda=9$

Figure 9: Slipstream behavior varing $\lambda$ and wind tunnel walls radius

On the contrary, the best prediction in all test sections is offered by the simulation that mimic the presence of the wind tunnel by a circular tunnel with $R=H / 2$ : in this case, in fact, both the far field value and the first peak in the axial velocity profiles are reasonably well captured. It can also be noted that the agreement degrades when moving downstream: this is probably due to the fact that the wind tunnel section in the experimental facility slowly grows in size in $x$ direction, and this last geometrical variation was not taken into account in the simplified tunnel form. Moreover, the observed difference between simulation and experiments are within the difference between the right and left profiles in the experiments, that can be taken as an estimation of the experimental uncertainties (given the symmetry of the problem). 


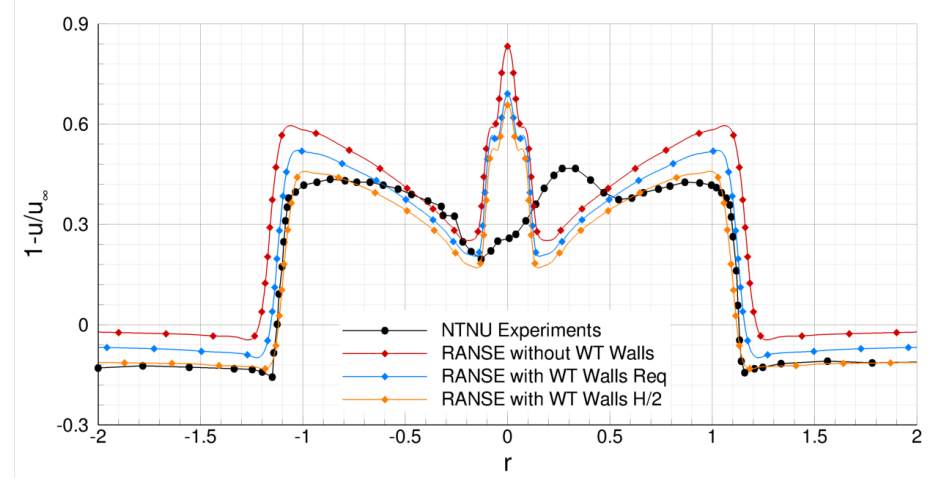

(a) $x / D=1$

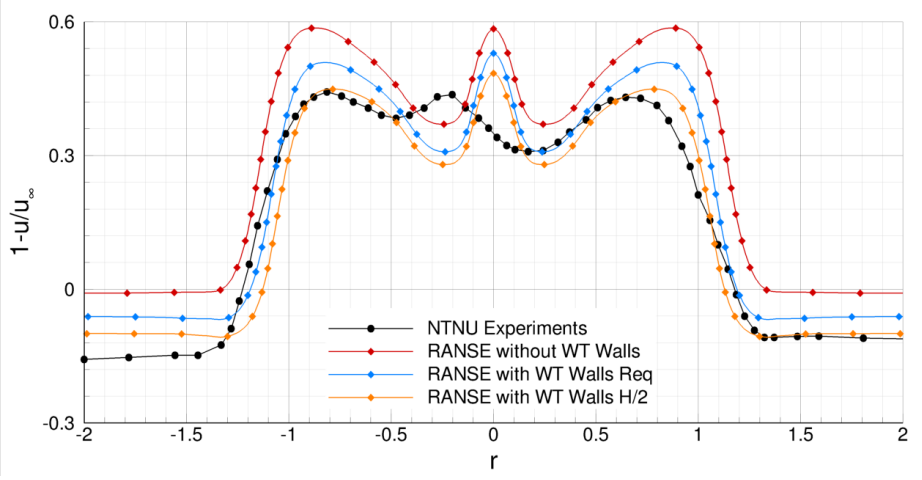

(b) $x / D=3$

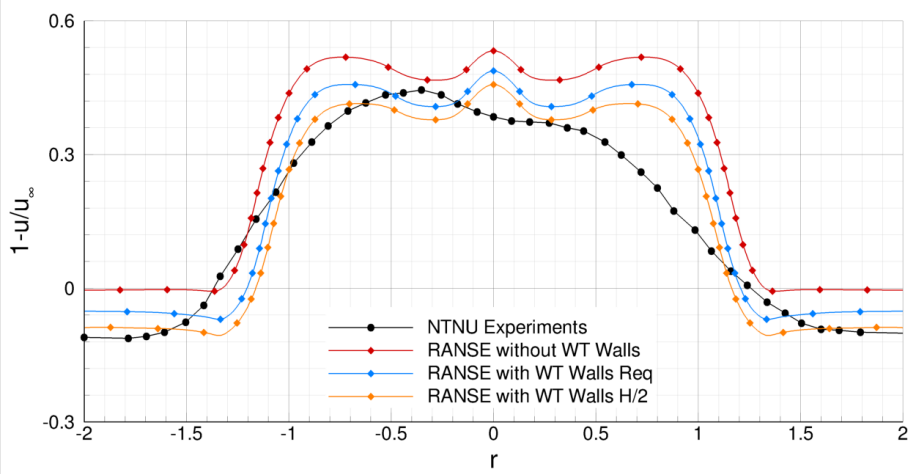

(c) $x / D=5$

Figure 10: Mean axial velocity defect along horizontal line for $\lambda=6, u$ being the axial velocity normalized by the undisturbed freestream inflow velocity

Figure 11 reports the mean velocity components along the radial profiles at the longitudinal section $x / D=1$ and $x / D=5$ for the three numerical campaigns. As for the slipstream geometrical details, it can be noted that all 
the velocity components are affected by the contraction of the wake enforced by the tunnel walls. The longitudinal and radial components are influenced by presence of the walls along almost all the radial section $0 \leq r / R \leq 2$, whereas the tangential component rapidly vanishes (together with the dependence on the wall position) as the blade tip is reached, i.e. for $r / R>1$. The same behaviour is observed for the downstream section $x / D=5$, where the effects of the walls proximity is obviously less relevant. Again, no information about this last data are available from the experiments. Nevertheless, on the basis of the comparison of axial velocity profiles previously shown, it is reasonable to consider the prediction with $R_{H / 2}$ more reliable than the other.

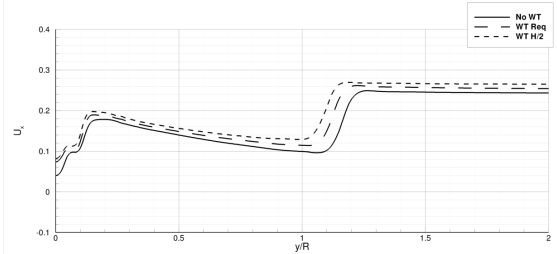

(a) $U_{x}, x / D=1$

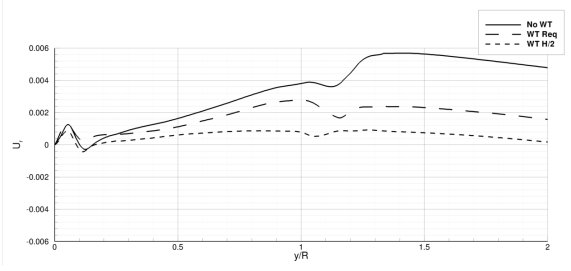

(c) $U_{r}, x / D=1$

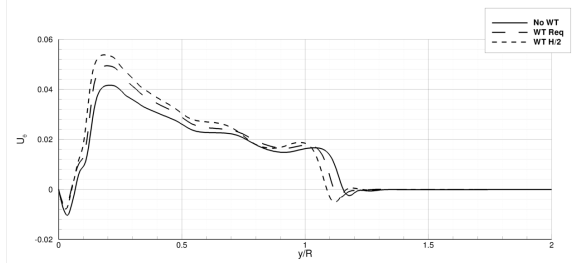

(e) $U_{\theta}, x / D=1$

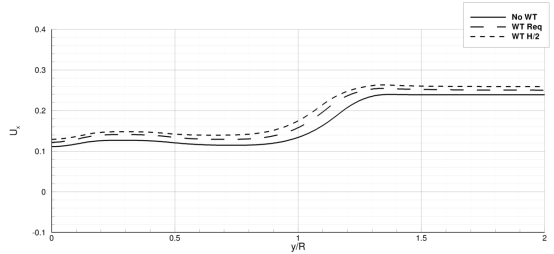

(b) $U_{x}, x / D=5$

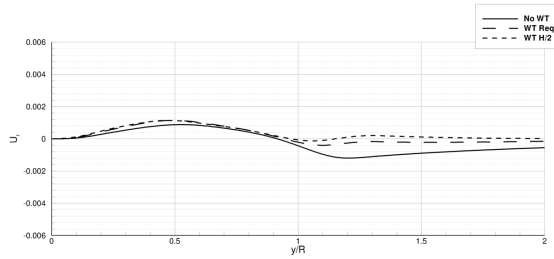

(d) $U_{r}, x / D=5$

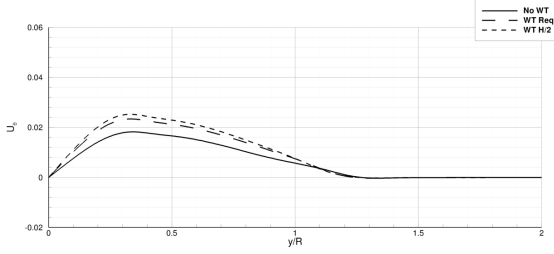

(f) $U_{\theta}, x / D=5$

Figure 11: Mean axial and radial velocity components for $\lambda=6, x / D=1,5$

\section{Concluding Remarks and Perspectives}

The possibility to simulate blockage effects in Reynolds average NavierStokes solvers by approximate tunnel geometry was investigated. In particular, 
the approximate geometry was chosen in order to retain the axi-symmetry of the isolated turbine simulation, so that the computation can be performed in the rotating frame where it is expected to be steady. The interest for such an equivalent geometry of course stems from the fact that a steady simulation in the rotating frame is far more efficient, from the computational point of view, than a costly and lengthy unsteady Navier-Stokes simulation in the absolute frame, that takes into account the real tunnel shape.

To this end, two cylindrical wind tunnels were considered: in the first, the cross section was chosen such that the area is the same as in the actual experiment; in the second, the position of the boundary was such that the blockage effects were supposed to be related to the smallest tunnel dimension.

The comparison was done for a test case were the correction factors computed according to the momentum theory are expected to fail, because of the relatively high blockage coefficient or because the turbine is highly loaded.

The obtained results proved that, unfortunately, there is not a unique equivalent section that allows to match both global coefficients and local flow features. In fact, the simulations with a section whose radius is such that the cross section is maintained seems to be the best choice as to the prediction of thrust and power coefficients, whereas the best prediction of the turbine slipstream is attained by a simulation with a closer wall. Nevertheless, the investigation proved that, if both information are required, a reasonable and efficient approach for all the correction factors can be obtained by only two steady simulations in the rotating frame, that are anyhow far more efficient than a complete unsteady computation.

\section{Acknowledgements}

The authors wish to thank the Department of Energy and Process Engineering of the Norwegian University of Science and Technology for providing all the geometrical data. In particular special thanks are due to Prof. Krogstad, P.A for the useful discussion and the experimental data.

We acknowledge the CINECA award under the ISCRA initiative, for the availability of high performance computing resources and support. 
This work has been partially supported by the Italian Ministry of Education, University and Research through the research project RITMARE.

\section{References}

[1] J. Sørensen, Aerodynamic Aspects of Wind Energy Conversion, Annual Review of Fluid Mechanics 43 (2011) 427-448.

[2] M. Hand, D. Simms, L. Fingersh, D. Jager, J. Cotrell, S. Schreck, S. Larwood, Unsteady Aerodynamics Experiment Phase VI: Wind Tunnel Test Configurations and Available Data Campaigns, Technical Report, National Renewable Energy Lab. (NREL), Golden, CO.(US), 2001.

[3] J. Schepers, H. Snel, Mexico project, Model experiments in controlled conditions, Technical Report, Energy Research Center of the Netherlands, 2007.

[4] H. Snel, J. Schepers, B. Montgomerie, The MEXICO project (Model Experiments in Controlled Conditions): the database and first results of data processing and interpretation, in: Journal of Physics: Conference Series, volume 75, IOP Publishing, p. 012014.

[5] P. Krogstad, M. Adaramola, Performance and near wake measurements of a model horizontal axis wind turbine, Wind Energy 15 (2012) 743-756.

[6] M. Adaramola, P. Krogstad, Experimental investigation of wake effects on wind turbine performance, Renewable Energy 36 (2011) 2078-2086.

[7] H. Glauert, The elements of aerofoil and airscrew theory, Cambridge University Press, 1983.

[8] A. Segalini, P. Inghels, Confinement effects in wind-turbine and propeller measurements, Journal of Fluid Mechanics 756 (2014) 110-129.

[9] M. J. Werle, Propeller Wall Blockage Performance Corrections, Journal of Propulsion and Power 27 (2010) 496-498.

[10] M. J. Werle, Wind Turbine Wall-Blockage Performance Corrections, Journal of Propulsion and Power 26 (2010) 1317-1321. 
[11] P. Krogstad, P. Eriksen, "Blind test" calculations of the performance and wake development for a model wind turbine, Renewable Energy 50 (2013) 325-333.

[12] P. Krogstad, J. Lund, An experimental and numerical study of the performance of a model turbine, Wind Energy 15 (2012) 443-457.

[13] D. Somers, The S825 and S826 Airfoils, Technical Report, National Renewable Energy Laboratory, 1617 Cole Boulevard, Golden, Colorado 804013393, US, 2005. Period of Performance: 1994-1995.

[14] A. Di Mascio, R. Broglia, B. Favini, A second order Godunov-type scheme for naval hydrodynamics, in: Godunov Methods: Theory and Applications, volume 26, Kluwer Academic/Plenum Publishers, 2001, pp. 253-261.

[15] A. Di Mascio, R. Broglia, R. Muscari, On the application of the singlephase level set method to naval hydrodynamic flows, Computers \& Fluids 36 (2007) 868-886.

[16] A. Di Mascio, R. Broglia, R. Muscari, Prediction of hydrodynamic coefficients of ship hulls by high-order Godunov-type methods, Journal of Marine Science and Technology 14 (2009) 19-29.

[17] R. Muscari, M. Felli, A. Di Mascio, Analysis of the Flow Past a Fully Appended Hull with Propellers by Computational and Experimental Fluid Dynamics, Journal of Fluids Engineering 133 (2011).

[18] G. Dubbioso, R. Muscari, A. Di Mascio, Analysis of the performances of a marine propeller operating in oblique flow, Computers \& Fluids 75 (2013) $86-102$.

[19] P. Spalart, S. Allmaras, A one-equation turbulence model for aerodynamic flows, La Recherce Aerospatiale 1 (1994) 5-21.

[20] C. Merkle, M. Athavale, Time-accurate unsteady incompressible flow algorithms based on artificial compressibility, in: AIAA $8^{t} h$ Computational Fluid Dynamics Conference, American Institute of Aeronautics and Astronautics, Honolulu, Hawaii (USA), pp. 397-407. 
[21] B. Favini, R. Broglia, A. Di Mascio, Multigrid acceleration of second-order ENO schemes from low subsonic to high supersonic flows, International Journal for Numerical Methods in Fluids 23 (1996) 589-606.

[22] R. Beam, R. Warming, An Implicit Factored Scheme for the Compressible Navier-Stokes Equations, AIAA Journal 16 (1978) 393-402.

[23] F. Pierella, P. Krogstad, L. Sætran, Blind test 2 calculations for two in-line model wind turbines where the downstream turbine operates at various rotational speeds, Renewable Energy 70 (2014) 62-77. Special issue on aerodynamics of offshore wind energy systems and wakes.

[24] F. Pierella, P. Eriksen, L. Sætran, P. Krogstad, Invitation to the 2012 "Blind test 2" Workshop Calculations for two wind turbines in line, Published by the Department of Energy and Process Engineering, NTNU, 2012.

[25] P. J. Roache, Quantification of Uncertainty in Computational Fluid Dynamics, Annual Review of Fluid Mechanics 29 (1997) 123-160.

[26] J. Jeong, F. Hussain, On the identification of a vortex, Journal of Fluid Mechanics 285 (1995) 69-94.

[27] R. Muscari, A. Di Mascio, R. Verzicco, Modelling of vortex dynamics in the wake of a marine propeller, Computers \& Fluids 73 (2013) 65-79.

[28] A. Di Mascio, R. Muscari, G. Dubbioso, On the wake dynamics of a propeller operating in drift, Journal of Fluid Mechanics 754 (2014) 263-307.

[29] P. Spalart, Detached-eddy simulation, Annual review of Fluid Mechanics 41 (2009) 181-202.

[30] P. Spalart, W. Jou, S. Allmaras, Comments on the feasibility of LES for wings, and on a hybrid RANS/LES approach, in: G. Press (Ed.), Advances in DNS/LES, First AFOSR International Conference on DNS/LES, Louisiana Tech University, Ruston, Louisiana, USA, pp. 137-47. 